\title{
THE WEINSTEIN CONJECTURE IN THE PRESENCE OF SUBMANIFOLDS HAVING A LEGENDRIAN FOLIATION
}

\author{
KLAUS NIEDERKRÜGER AND ANA RECHTMAN
}

\begin{abstract}
Helmut Hofer introduced in '93 a novel technique based on holomorphic curves to prove the Weinstein conjecture. Among the cases where these methods apply are all contact 3 -manifolds $(M, \xi)$ with $\pi_{2}(M) \neq 0$. We modify Hofer's argument to prove the Weinstein conjecture for some examples of higher dimensional contact manifolds. In particular, we are able to show that the connected sum with a real projective space always has a closed contractible Reeb orbit.
\end{abstract}

\section{INTRODUCTION}

Let $(M, \xi)$ be a contact manifold with contact form $\alpha$. The associated Reeb field $R_{\alpha}$ is the unique vector field that satisfies the equations

$$
\alpha\left(R_{\alpha}\right)=1 \quad \text { and } \quad \iota_{R_{\alpha}} d \alpha=0
$$

everywhere.

Weinstein conjecture. Let $(M, \xi)$ be a closed contact manifold, and choose any contact form $\alpha$ with $\xi=\operatorname{ker} \alpha$. The Reeb field $R_{\alpha}$ associated to $\alpha$ always has a closed orbit.

In his seminal paper Hof93, Helmut Hofer found a strong relation between the dynamics of the Reeb field and holomorphic curves in symplectizations. Initially Hofer proved the Weinstein conjecture using these methods in three cases, namely the conjecture holds for a closed contact 3 -manifold $(M, \xi)$, if $M$ is diffeomorphic to $\mathbb{S}^{3}$, if $\xi$ is overtwisted or if $\pi_{2}(M) \neq 0$.

A generalization of the second case to higher dimensions has been achieved in [AH09] for contact structures that have a Plastikstufe. We will try to generalize the third one. In order to justify our hypothesis, let us recall the key steps in Hofer's proof. The non-triviality of the second homotopy group combined with the assumption that the contact structure is tight, allow us to find a noncontractible embedded sphere whose characteristic foliation has only two elliptic singularities and no closed leaves. Near the singularities, an explicit Bishop family of holomorphic disks can be constructed, and it can be proved that the disks produce a finite energy plane. The existence of a finite energy plane in a symplectization of the manifold implies the existence of a contractible periodic Reeb orbit.

Our generalization of Hofer's theorem for contact $(2 n+1)$-manifolds replaces the 2 -sphere by an embedded $(n+1)$-submanifold such that the contact structure restricts to an open book decomposition. Following Hofer's ideas, we will prove that if such a submanifold represents a non-trivial homology class then there exists a periodic contractible Reeb orbit.

Among the examples where we are able to find such submanifolds, are the connected sum of any contact manifold $M$ with

(a) the projetive space with its standard contact structure

(b) certain subcritically fillable manifolds as for example $\mathbb{S}^{n} \times \mathbb{S}^{n+1}$ or $\mathbb{T}^{n} \times \mathbb{S}^{n+1}$.

Acknowledgments. Several people helped us writing this article: In particular we would like to thank Chris Wendl for spotting a mistake in a preliminary version and Janko Latschev for solving this problem. We also received helpful comments by Emmanuel Giroux and Leonid Polterovich.

The first author would like to thank the University of Chicago, the MSRI, and the CCCI for partially funding his stay in Chicago, where the initial ideas of this paper have been developed.

The second author would like to thank the CONACyT for her postdoctoral fellowship. 


\section{THE MAIN CRITERIA}

Definition. An open book decomposition $(\vartheta, B)$ of a manifold $N$ consists of

(i) a proper codimension 2 submanifold $B \subset N$ that has a tubular neighborhood that is diffeomorphic to $B \times \mathbb{C}$, and

(ii) a proper fibration $\vartheta:(N \backslash B) \rightarrow \mathbb{S}^{1}$ such that the map $\vartheta$ agrees on the neighborhood $B \times \mathbb{C}$ with the angular coordinate $e^{i \varphi}$ of the $\mathbb{C}$-factor.

The submanifold $B$ is called the binding, and the fibers of $\vartheta$ are called the pages of the open book. From the definition it follows that the closure of a page $P$ in $N$ is a compact manifold with boundary $B$.

Remark 1. Open book decompositions are typically only studied on closed manifolds, in which case the binding is also a closed manifold. In this article, we will first restrict to closed manifolds, but then we will study closed manifolds whose universal cover admits an open book decomposition, and we do not want to suppose that the universal cover itself is a closed manifold.

Assume $(M, \xi)$ is a contact $(2 n+1)$-manifold. A submanifold $N \hookrightarrow M$ is called maximally foliated by $\xi$ if $\operatorname{dim} N=n+1$, and if the intersection $\xi \cap T N$ defines a singular foliation on $N$. The regular leaves of such a foliation are locally Legendrian submanifolds.

Definition. In the situation above, we say that $N \hookrightarrow(M, \xi)$ carries a Legendrian open book, if the maximal foliation on $N$ defines an open book decomposition of $N$, i.e., the singular set $\left\{p \in N \mid T_{p} N \subset \xi_{p}\right\}$ is the binding of an open book on $N$, and each regular leaf of the foliation corresponds to a page of the open book.

The following notion is extensively studied in $\underline{\mathrm{MNW}}$ as a filling obstruction, here we will only use it as a sufficient condition for the existence of a closed contractible Reeb orbit.

Definition. Let $N$ be a compact submanifold of $(M, \xi)$ that is maximally foliated by $\xi$, and has non-empty boundary $\partial N$ that can be written as a product manifold $\partial N \cong \mathbb{S}^{1} \times L$. We say that $N$ carries a Legendrian open book with boundary, if the following conditions are satisfied by the foliation:

(i) The singular set is the union of the boundary $\partial N$ and a closed (not necessarily connected) codimension 2 submanifold $B \subset N \backslash \partial N$ with trivial normal bundle.

(ii) There exists a submersion

$$
\vartheta: N \backslash B \rightarrow \mathbb{S}^{1}
$$

that restricts on $\partial N \cong \mathbb{S}^{1} \times L$ to the projection onto the first factor.

(iii) The regular leaves of the Legendrian foliation $\xi \cap T N$ are the fibers of $\vartheta$ intersected with the interior of $N$.

(iv) The neighborhood of $B$ has a trivialization $B \times \mathbb{C}$ for which the angular coordinate $e^{i \varphi}$ on $\mathbb{C}$ agrees with the map $\vartheta$.

Remark 2. There are two common definitions of the overtwisted disk; according to one version the boundary is a regular compact leaf of the foliation, but there is a second version where the foliation is singular along the boundary of the disk. This second definition is an example of a Legendrian open book with boundary. By a small perturbation it is always possible to move from one version to the other one, so that both definitions are equivalent. Similarly, it is possible to deform a plastikstufe to obtain a Legendrian open book with boundary, so that the definition above includes $P S$-overtwisted manifolds.

Theorem 1. Let $(M, \xi)$ be a closed contact manifold, and let $N$ be a compact submanifold.

(i) If $\xi$ induces a Legendrian open book on $N$ (without boundary), and if $\xi$ admits a contact form $\alpha$ without closed contractible Reeb orbits, then it follows that $N$ represents the trivial homology class in $H_{n+1}\left(M, \mathbb{Z}_{2}\right)$.

(ii) If $\xi$ induces a Legendrian open book with boundary on $N$, then every contact form $\alpha$ on $(M, \xi)$ has a closed contractible Reeb orbit. 
Remark 3. In the situation of Theorem 1. (ii), it also follows that $(M, \xi)$ does not admit a (semipositive) strong symplectic filling in general, and under some cohomological condition it even excludes the existence of a weak filling. The proof of this fact is given in [MNW]. We will call such a contact structure $P S$-overtwisted.

Remark 4. It should be possible to strengthen the conclusions of the theorem. For example, if both $N$ and the moduli space used in the proof of (i) are orientable, the coefficients for the homology group can be taken in $\mathbb{Z}$.

Proof of Theorem 1. Following Hofer's idea for 3-manifolds, we will study a moduli space of holomorphic disks in the symplectization of $(M, \alpha)$. To prove (i), we will then show that the union of these holomorphic disks represents a chain in $M$ whose boundary is homologous to the submanifold $N$. The proof of (ii) is based on a contradiction to Gromov compactness as in [AH09], and we will only discuss it briefly at the end.

(i) First, we have to choose a suitable almost complex structure $J$ on the symplectization

$$
\left(\mathbb{R} \times M, d\left(e^{t} \alpha\right)\right) .
$$

We embed $(M, \alpha)$ as the 0 -level set $\{0\} \times M$, and define $J$ first in a neighborhood of the binding $B$ in $\{0\} \times N$, before extending it over all of $\mathbb{R} \times M$. It was shown in [Nie06, Section 3] that the germ of the contact form in a neighborhood of $B$ is completely determined by the foliation on $N$, or said otherwise, there is a neighborhood $U$ around the binding $B$ that is strictly contactomorphic to a neighborhood $\widetilde{U}$ of the 0 -section in

$$
\left(\mathbb{R}^{3} \times T^{*} B, d z+\frac{1}{2}(x d y-y d x)+\lambda_{\text {can }}\right),
$$

where $(x, y, z)$ are the standard coordinates on $\mathbb{R}^{3}$, and $\lambda_{\text {can }}$ is the canonical 1 -form on $T^{*} B$. The set $U \cap N$ corresponds in this model to the intersection of $\widetilde{U}$ with the submanifold $\{(x, y, 0)\} \times B$.

We will now study the following model for the symplectization of $U$ : Let $W_{1}=\mathbb{C}^{2}$ be the Stein manifold with standard complex, and symplectic structures, and with the plurisubharmonic function $h_{1}\left(z_{1}, z_{2}\right)=\left|z_{1}\right|^{2}+\left|z_{2}\right|^{2}$. To find a Weinstein structure on $T^{*} B$ choose a Riemannian metric $g$ on the binding $B$, then the cotangent bundle $W_{2}=T^{*} B$ carries an induced Riemannian metric $\widetilde{g}$, and an exact symplectic structure $d \lambda_{\text {can }}$ given by the differential of the canonical 1-form $\lambda_{\text {can }}:=-\mathbf{p} d \mathbf{q}$. There is a unique almost complex structure $J_{g}$ on $W_{2}$ that is compatible with $d \lambda_{\text {can }}$ and with the metric $\widetilde{g}$. The function $h_{2}(\mathbf{q}, \mathbf{p})=\|\mathbf{p}\|^{2} / 2$ is $J_{g}$-plurisubharmonic and satisfies $d h_{2} \circ J_{g}=-\lambda_{\text {can }}$ (see also [Nie06, Appendix B]).

The product manifold $W=W_{1} \times W_{2}=\mathbb{C}^{2} \times T^{*} B$ is a Weinstein manifold with almost complex structure $J^{\prime}=i \oplus J_{g}$, and plurisubharmonic function $h=h_{1}+h_{2}$. Its contact type boundary $M^{\prime}:=h^{-1}(1)$ contains the submanifold

$$
\left\{\left(\sqrt{1-|z|^{2}}, z ; \mathbf{q}, \mathbf{0}\right)|| z \mid<\varepsilon\right\} \cong \mathbb{D}^{2} \times B .
$$

The natural contact structure $\operatorname{ker}\left(d h \circ J^{\prime}\right)$ on $M^{\prime}$ induces a singular foliation on this submanifold that is diffeomorphic to the neighborhood of the binding of an open book, so that in fact the neighborhood of this submanifold in $W$ is symplectomorphic to a neighborhood of $\{0\} \times B$ in the symplectization, and the plurisubharmonic function $h$ coincides with $e^{t}$ on $\mathbb{R} \times M$.

The pull-back of $J^{\prime}=i \oplus J_{g}$ to the symplectization defines thus an almost complex structure in a neighborhood of the binding $\{0\} \times B$ in $\mathbb{R} \times M$, which we can easily extend to an almost complex structure on $(-\varepsilon, \varepsilon) \times M$ that is compatible with the symplectic form $d\left(e^{t} \alpha\right)$, and for which $d t \circ J=\alpha$. Unfortunately this almost complex structure is not $t$-invariant, but we can extend $J$ to an almost complex structure that is tamed by $d\left(e^{t} \alpha\right)$ everywhere, restricts to $\xi$, and is $t$-invariant below a certain level set $\{-C\} \times M$ in the symplectization.

With the chosen almost complex structure $J$, it is easy to explicitly write down a Bishop family of holomorphic disks in a neighborhood of $\{0\} \times B$, and to use an intersection argument to exclude the existence of other holomorphic disks in this neighborhood. Namely, the Bishop family will be given in the model $\mathbb{C}^{2} \times T^{*} B$ by the intersection of the 2 -planes

$$
E_{t_{0}, \mathbf{q}_{0}}:=\left\{\left(t_{0}, z ; \mathbf{q}_{0}, \mathbf{0}\right) \mid \mathbf{q}_{0} \in B, t_{0}<1, z \in \mathbb{C}\right\}
$$


with $h^{-1}((1-\varepsilon, 1])$. The result gives for every point $\mathbf{q}_{0}$ of the binding $B$ a 1 -dimensional family of round disks attached with their boundary to the foliated submanifold. The radius of the disk decreases as $t_{0} \rightarrow 1$, and in the limit the disks collapse to the point $\mathbf{q}_{0} \in B$. All of the disks are pairwise disjoint, and if we look at the space of parameterized disks, we obtain thus a smooth $(n+3)$-dimensional manifold.

To exclude the existence of other disks close to the binding, we use an intersection argument with the local foliation given by $\left(i \oplus J_{g}\right)$-holomorphic codimension 2 submanifolds

$$
S_{z_{0}}:=\left\{\left(z_{0}, z\right) \mid z \in \mathbb{C}\right\} \times T^{*} B
$$

with $\operatorname{Re} z_{0}<1$. For more details see [Nie06, Section 3].

We will now look at the moduli space of holomorphic disks given as follows: Denote $N \backslash B$ by $\stackrel{\circ}{N}$, and let $\widetilde{\mathcal{M}}$ be the space of all $J$-holomorphic maps

$$
u:\left(\mathbb{D}^{2}, \partial \mathbb{D}^{2}\right) \rightarrow((-\infty, 0] \times M,\{0\} \times \stackrel{\circ}{N}),
$$

whose boundary $u\left(\partial \mathbb{D}^{2}\right)$ intersects every page of the open book on $N$ exactly once. For simplicity we will restrict to the component of $\widetilde{\mathcal{M}}$ that contains the Bishop family (for every component of the binding there is an independent Bishop family, but one result of our assumptions will be that all these families lie in the same component of the moduli space).

Before producing a moduli space by taking a quotient of $\widetilde{\mathcal{M}}$, we will briefly discuss Gromov compactness. We claim that there is a uniform energy bound for all curves $u \in \widetilde{\mathcal{M}}$. The energy of a holomorphic curve $u$ in a symplectization is defined as

$$
E_{\alpha}(u):=\sup _{\varphi \in \mathcal{F}} \int_{u} d(\varphi \alpha)
$$

where $\mathcal{F}$ is the set of smooth functions $\varphi: \mathbb{R} \rightarrow[0,1]$ with $\varphi^{\prime} \geq 0$. Here we identify $\mathbb{R}$ with the $\mathbb{R}$-factor of the symplectization.

Using Stokes' Theorem, we easily obtain for any holomorphic disk $u \in \widetilde{\mathcal{M}}$ that

$$
E_{\alpha}(u)=\int_{\partial u} \alpha .
$$

There is a continuous function $f: N \rightarrow[0, \infty)$ such that $\left.\alpha\right|_{T N}=f d \vartheta$, where $\vartheta: N \backslash B \rightarrow \mathbb{S}^{1}$ is the fibration of the open book, and because the boundary of the curves $u\left(\partial \mathbb{D}^{2}\right)$ crosses every page of the open book on $N$ exactly once, we obtain the energy bound

$$
E_{\alpha}(u) \leq 2 \pi \max _{x \in N} f(x),
$$

proving the claim.

Let $\left(u_{k}\right)_{k} \subset \widetilde{\mathcal{M}}$ be a sequence of holomorphic maps. The only disks that may intersect a small neighborhood of the binding $\{0\} \times B$ are the ones that lie in the Bishop family, and hence we will assume that all maps $u_{k}$ stay at finite distance from the binding $\{0\} \times B$, because otherwise it follows that the $u_{k}$ collapse to a point in $B$.

Proposition 1. Let $\left(u_{k_{n}}\right)_{n}$ be a sequence of holomorphic maps whose image is bounded away from $\{0\} \times B$. There there is a subsequence $\left(u_{k_{n}}\right)_{n}$ and a family of biholomorphisms $\varphi_{n} \in \operatorname{Aut}\left(\mathbb{D}^{2}\right)$, such that the reparameterized maps $\left(u_{k_{n}} \circ \varphi_{n}\right)_{n}$ converge uniformly in $C^{\infty}$ to a map $u_{\infty} \in \widetilde{\mathcal{M}}$.

Proof. Assume the conclusion is false, then the gradient of the reparameterized sequence is blowing up, and this would either lead to the existence of a holomorphic sphere, a finite energy plane, or a disk bubbling off. Symplectizations never contain holomorphic spheres, and since by our assumption $(M, \alpha)$ does not have closed contractible Reeb orbits, we also have excluded the existence of finite energy planes. Finally, bubbling of disks is not allowed because the maps in $\widetilde{\mathcal{M}}$ cross every page of the open book on $N$ exactly once, and this implies that the boundary of the disks are undecomposable. 
With the limit behavior of the maps in $\widetilde{\mathcal{M}}$ understood, we will now study the moduli space

$$
\mathcal{M}:=\widetilde{\mathcal{M}} \times \mathbb{D}^{2} / \sim
$$

where we identify pairs $(u, z),\left(u^{\prime}, z^{\prime}\right) \in \widetilde{\mathcal{M}} \times \mathbb{D}^{2}$, if and only if there is a Möbius transformation $\varphi \in \operatorname{Aut}\left(\mathbb{D}^{2}\right)$ such that $(u, z)=\left(u^{\prime} \circ \varphi^{-1}, \varphi\left(z^{\prime}\right)\right)$. Note that the action of $\operatorname{Aut}\left(\mathbb{D}^{2}\right)$ on $\widetilde{\mathcal{M}}$ is proper and free because every map $u \in \widetilde{\mathcal{M}}$ is injective along its boundary, and the identity is the only biholomorphism of $\mathbb{D}^{2}$ that keeps the boundary of the disk pointwise fixed. It follows that $\mathcal{M}$ is a non-compact smooth $(n+2)$-dimensional manifold with boundary. The boundary corresponds to equivalence classes $[u, z] \in \mathcal{M}$ with $z \in \partial \mathbb{D}^{2}$. Proposition 1 above allows to understand that the compactification of $\mathcal{M}$ is in fact a smooth compact manifold with boundary: If $\left(\left[u_{k}, z_{k}\right]\right)_{k}$ is a sequence of elements in $\mathcal{M}$, and if the image of the maps $u_{k}$ stays at a finite distance from the binding $\{0\} \times B$, then we know that there is a subsequence $\left(\left[u_{k_{n}}, z_{k_{n}}\right]\right)_{n}$ and a family of reparameterizations $\varphi_{n} \in \operatorname{Aut}\left(\mathbb{D}^{2}\right)$ such that $u_{k_{n}} \circ \varphi_{n}^{-1}$ converges locally uniformly to a map $u_{\infty} \in \widetilde{\mathcal{M}}$. The subsequence $\left(\left[u_{k_{n}} \circ \varphi_{n}^{-1}, \varphi_{n}\left(z_{k_{n}}\right)\right]\right)_{n}$ contains a further subsequence that converges to a proper element $\left[u_{\infty}, z_{\infty}\right]$ of the moduli space $\mathcal{M}$.

If the image of a map $u_{k}$ intersects a small neighborhood $U$ of the binding in the symplectization, then it is up to reparameterization an element of the Bishop family. Thus, when the image of the maps $u_{k}$ gets close to the binding $\{0\} \times B$, we can find a subsequence $\left(\left[u_{k_{n}}, z_{k_{n}}\right]\right)_{n}$ such that all the $u_{k_{n}}$ lie in the Bishop family. Here, we can describe $\mathcal{M}$ and its closure explicitly. The $E_{t_{0}, \mathbf{q}_{0}}$-planes are all pairwise disjoint, hence we have that there is exactly one disk $[u, z] \in \mathcal{M}$ with $u(z)=p$ for every $p$ in the symplectization lying in the image of the Bishop family $\{(t, z ; \mathbf{q}, \mathbf{0}) \mid \mathbf{q} \in B, t<$ $\left.1, z \in \mathbb{C},|z|^{2} \leq 1-t^{2}\right\}$. Then the compactification of the Bishop family is naturally diffeomorphic to the smooth manifold with boundary

$$
\left\{\left.(t, z ; \mathbf{q}, \mathbf{0})|\mathbf{q} \in B, t \leq 1, z \in \mathbb{C},| z\right|^{2} \leq 1-t^{2}\right\} .
$$

There is a well-defined smooth evaluation map

$$
\text { ev: } \overline{\mathcal{M}} \rightarrow \mathbb{R} \times M, \quad[u, z] \mapsto u(z)
$$

from the compactification of the moduli space into the symplectization.

Definition. The degree $\operatorname{deg} f \in \mathbb{Z}_{2}$ of a continuous map $f: X \rightarrow Y$ between two closed $n^{-}$ manifolds $X$ and $Y$ is defined as the element $A \in \mathbb{Z}_{2}$ such that $f_{\#}[X]=A[Y] \in H_{n}\left(Y, \mathbb{Z}_{2}\right)$.

For smooth maps it is easy to compute $\operatorname{deg} f$, because it suffices to take a regular value $y \in Y$ of $f$, and count Eps66

$$
\operatorname{deg} f=\# f^{-1}(y) \bmod 2 .
$$

Hence, it follows immediately that the restriction of the evaluation map to the boundary $\partial \overline{\mathcal{M}}$ of the moduli space is a smooth map

$$
\left.\mathrm{ev}\right|_{\partial \overline{\mathcal{M}}}: \partial \overline{\mathcal{M}} \rightarrow\{0\} \times N
$$

of degree 1 (as can be easily seen by using that close to the binding $\{0\} \times B$ there is for every $p \in\{0\} \times N$ a unique disk $[u, z] \in \partial \overline{\mathcal{M}}$ with $u(z)=p)$. In particular by combining the trivial identity

$$
\mathrm{ev} \circ \iota_{\partial \overline{\mathcal{M}}}=\left.\iota_{N} \circ \mathrm{ev}\right|_{\partial \overline{\mathcal{M}}}
$$

for the standard inclusions $\iota_{\partial \overline{\mathcal{M}}}: \partial \overline{\mathcal{M}} \hookrightarrow \overline{\mathcal{M}}$ and $\iota_{N}: N \hookrightarrow \mathbb{R} \times M$, with the fact that $\partial \overline{\mathcal{M}}$ is nullhomologous in $H_{n+1}\left(\overline{\mathcal{M}}, \mathbb{Z}_{2}\right)$, and using that ev $\circ \iota_{\partial \overline{\mathcal{M}}}$ induces the trivial map on $H_{n+1}\left(\partial \overline{\mathcal{M}}, \mathbb{Z}_{2}\right)$, we obtain that

$$
\left(\iota_{N}\right)_{\#}: H_{n+1}\left(N, \mathbb{Z}_{2}\right) \rightarrow H_{n+1}\left(M, \mathbb{Z}_{2}\right)
$$

vanishes, because $\left(\left.\mathrm{ev}\right|_{\partial \overline{\mathcal{M}}}\right)_{\#}$ is an isomorphism. It follows that $N$ represents a trivial $(n+1)$-class in $H_{n+1}\left(M, \mathbb{Z}_{2}\right)$ as we wanted to show.

(ii) If $N$ carries a Legendrian open book with boundary, we will proceed as follows: Choose close to the binding $\{0\} \times B$ on the symplectization the almost complex structure described above that allows us to find the Bishop family of holomorphic disks. 
In [NP10, Section 5.3], it was shown that we can find a specific almost complex structure on a neighborhood of the boundary $\{0\} \times \partial N \cong \mathbb{S}^{1} \times L$ that prevents any holomorphic disk to enter this area. After choosing these two almost complex structures, close to the binding $B$ and to the boundary $\partial N$, extend them to a global almost complex structure $J$ on $\mathbb{R} \times M$ that is compatible with the symplectic form $d\left(e^{t} \alpha\right)$, and for which $d t \circ J=\alpha$. Additionally, we require $J$ to be $t$-invariant below a certain level set $\{-C\} \times M$ in the symplectization.

Denote now $N \backslash(B \cup \partial N)$ by $\stackrel{\circ}{N}$, and study the space $\widetilde{\mathcal{M}}$ of $J$-holomorphic maps

$$
u:\left(\mathbb{D}^{2}, \partial \mathbb{D}^{2}\right) \rightarrow((-\infty, 0] \times M,\{0\} \times \stackrel{\circ}{N}),
$$

whose boundaries $u\left(\partial \mathbb{D}^{2}\right)$ transverse every page of the open book on $N$ exactly once. If we assume that $(M, \alpha)$ does not have any contractible periodic Reeb orbits, then the compactness argument for sequences in $\widetilde{\mathcal{M}}$ works as above, because there is an area around $\partial N$ where no holomorphic curves are allowed to enter.

The moduli space, we will study now is given by

$$
\mathcal{M}:=\widetilde{\mathcal{M}} \times \mathbb{S}^{1} / \sim
$$

where we identify pairs $(u, z),\left(u^{\prime}, z^{\prime}\right) \in \widetilde{\mathcal{M}} \times \mathbb{S}^{1}$, if and only if there is a Möbius transformation $\varphi \in \operatorname{Aut}\left(\mathbb{D}^{2}\right)$ such that $(u, z)=\left(u^{\prime} \circ \varphi^{-1}, \varphi\left(z^{\prime}\right)\right)$. By the arguments above, $\mathcal{M}$ is a smooth $(n+1)$-dimensional manifold with a smooth evaluation map

$$
\mathrm{ev}: \mathcal{M} \rightarrow\{0\} \times N, \quad[u, z] \mapsto u(z) .
$$

If we choose a generic (differentiable) path $\gamma:[0,1] \rightarrow N$ that connects a binding component of $B$ with a component of the boundary $\partial N$, and is such that $\gamma(] 0,1[) \subset \stackrel{\circ}{N}$, then the evaluation map is transverse to $\gamma$. The pre-image $\mathrm{ev}^{-1}(\gamma)$ is a non-empty 1-dimensional smooth submanifold of $\mathcal{M}$. We only consider the component $\mathcal{M}_{0}$ of ev ${ }^{-1}(\gamma)$ that contains elements of the Bishop family.

The closure of the submanifold $\mathcal{M}_{0}$ has one end that corresponds to the disks that collapse to a point on the binding, and so $\mathcal{M}_{0}$ cannot be a circle, but must be instead an interval. The other end of the interval exists by Gromov compactness, but by our assumptions this limit curve will be a regular element of $\mathcal{M}_{0}$, so that in fact it is not the end of the interval leading to a contradiction, which implies the existence of a closed contractible Reeb orbit.

We can generalize Theorem 1 by changing open books to covered open books, let us start with the definition.

Definition. Let $N$ be a closed manifold with universal cover $\pi: \widetilde{N} \rightarrow N$. A pair $(\vartheta, B)$ consisting of a closed codimension 2 submanifold $B$ of $N$, and a proper fibration $\vartheta:(N \backslash B) \rightarrow \mathbb{S}^{1}$, is called a $k$-fold covered open book decomposition of $N$ if it induces an open book decomposition on the universal cover $\widetilde{N}$. More precisely, we require that there is an open book decomposition $(\widetilde{\vartheta}, \widetilde{B})$ on $\widetilde{N}$, where the binding $\widetilde{B}$ is $\pi^{-1}(B)$, and where the fibration $\widetilde{\vartheta}: \widetilde{N} \backslash \widetilde{B} \rightarrow \mathbb{S}^{1}$ commutes with $\pi$ and $z \mapsto z^{k}$ according to the following diagram:

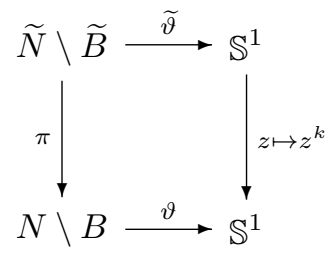

Definition. Accordingly we say that a maximally foliated submanifold $N$ of a contact manifold carries a Legendrian covered open book, if the maximal foliation on $N$ defines a covered open book decomposition of $N$.

If $N$ is a maximally foliated compact submanifold with boundary in a contact manifold $(M, \xi)$, then we say that $\xi$ induces a Legendrian covered open book with boundary if the foliation on the interior of $N$ defines a covered open book, and if it satisfies close to the boundary the same conditions as a proper Legendrian open book with boundary. 
Example 1. Note that a proper open book decomposition $(\vartheta, B)$ of a manifold $N$ is a 1 -fold covered open book decomposition, as the open book on $\widetilde{N}$ will be given by $\widetilde{\vartheta}=\vartheta \circ \pi$, and $\widetilde{B}=\pi^{-1}(B)$.

But this of course does not imply that $\widetilde{N}$ is a 1 -fold cover of $N$, as the following example shows: The standard open book decomposition on $\mathbb{S}^{2}$ (see Fig. 1 induces in an obvious way an open book decomposition on the manifold $\mathbb{S}^{1} \times \mathbb{S}^{2}$, and its universal cover $\mathbb{R} \times \mathbb{S}^{2}$.

Example 2. The unit sphere $\mathbb{S}^{n-1}=\left\{\left(x_{1}, \ldots, x_{n}\right) \in \mathbb{R}^{n} \mid x_{1}^{2}+\cdots+x_{n}^{2}=1\right\}$ admits an open book with binding $B=\left\{\left(x_{1}, \ldots, x_{n}\right) \in \mathbb{S}^{n-1} \mid x_{1}=x_{2}=0\right\}$, and fibration map

$$
\vartheta: \mathbb{S}^{n-1} \backslash B \rightarrow \mathbb{S}^{1}, \quad\left(x_{1}, \ldots, x_{n}\right) \mapsto \frac{\left(x_{1}, x_{2}\right)}{\sqrt{x_{1}^{2}+x_{2}^{2}}} .
$$

The binding is an $(n-3)$-sphere, and the pages are $(n-2)$-balls (see Fig. 1 ).

The real projective space $\mathbb{R P}^{n-1}$ can be obtained as the quotient of the unit sphere $\mathbb{S}^{n-1}$ by the antipodal map

$$
A: \mathbb{S}^{n-1} \rightarrow \mathbb{S}^{n-1}, \quad\left(x_{1}, \ldots, x_{n}\right) \mapsto\left(-x_{1}, \ldots,-x_{n}\right) .
$$

The open book on $\mathbb{S}^{n-1}$ described above projects onto a covered open book of $\mathbb{R P}^{n-1}$ with binding $B^{\prime}=\left\{\left[0: 0: x_{3}: \cdots: x_{n}\right] \in \mathbb{R} \mathrm{P}^{n-1}\right\} \cong \mathbb{R} \mathrm{P}^{n-3}$, and fibration map

$$
\vartheta^{\prime}: \mathbb{R P}^{n-1} \backslash B^{\prime} \rightarrow \mathbb{S}^{1}, \quad\left[x_{1}: \cdots: x_{n}\right] \mapsto \frac{\left(x_{1}^{2}-x_{2}^{2}, 2 x_{1} x_{2}\right)}{x_{1}^{2}+x_{2}^{2}},
$$

which is induced by the square of $\vartheta$. The pages of this open book are still $(n-2)-$ balls, but the monodromy is the antipodal map, and going around the binding once corresponds to crossing all pages twice (see Fig. 2). This way we obtain a 2 -fold covered open book of $\mathbb{R P}^{n-1}$ that is not a proper open book decomposition.

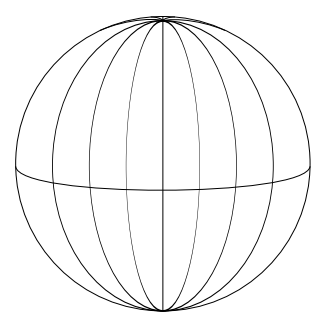

FiguRE 1. The standard open book on the 2-sphere.

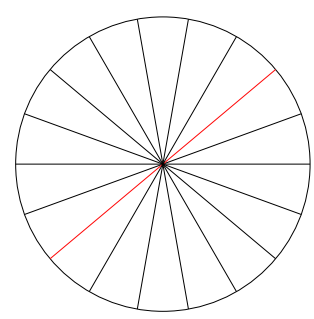

FiguRE 2. The induced covered open book on $\mathbb{R P}^{2}$. The boundary of the disk is identified under the antipodal map, so that for example the line drawn in red represents a single page that touches the binding at both of its boundaries.

Definition. Let $N$ be a closed submanifold of a contact manifold $(M, \xi)$, and assume that $\xi$ induces a Legendrian $k$-fold covered open book on $N$. We say that $N$ is nucleation free, if every loop $\gamma: \mathbb{S}^{1} \rightarrow N \backslash B$ that projects via $\vartheta: N \backslash B \rightarrow \mathbb{S}^{1}$ to a generator of $\pi_{1}\left(\mathbb{S}^{1}\right)$, represents in $\pi_{1}(M)$ an element that is at least of order $k$. 
Remark 5. The reason for our definition of nucleation free is that it excludes bubbling of certain holomorphic disks in the symplectization $\mathbb{R} \times M$. The boundary of every non-constant holomorphic disk $u$ that is attached with $\partial u$ to the submanifold $\{0\} \times N$ will always have positive transverse intersections with the pages of the covered open book. Since $\partial u$ is also clearly contractible in $M$, it follows that $\vartheta(\partial u) \subset \mathbb{S}^{1}$ will make a (positive) multiple of $k$ turns in the covered open book.

If we then choose a sequence of holomorphic disks $\left(u_{n}\right)_{n}$ such that each one intersects every page of the open book exactly $k$ times, the limit curve of $\left(u_{n}\right)_{n}$ cannot decompose into several non-constant holomorphic disks $v_{1}, \cdots, v_{N}$, because the boundary of these curves would describe loops in $N \backslash B$ that are contractible in $M$, but that make strictly less than $k$ turns in the covered open book.

Theorem 2. Let $(M, \xi)$ be a closed contact manifold, and let $N$ be a compact submanifold.

(i) If $\xi$ admits a contact form $\alpha$ without closed contractible Reeb orbits, and if it induces a Legendrian covered open book on $N$ that is nucleation free, then $N$ represents the trivial homology class in $H_{n+1}\left(M, \mathbb{Z}_{2}\right)$.

(ii) If $\xi$ induces on $N$ a Legendrian covered open book with boundary that is nucleation free, then every contact form $\alpha$ of $(M, \xi)$ has a closed contractible Reeb orbit.

Remark 6. Note that the conditions in Theorem 2.(ii) do not imply the non-fillability of $M$. Nonetheless, it implies that there is a loop in $N \backslash B$ that projects via $\vartheta$ onto a positive generator of $\mathbb{S}^{1}$, and represents in the filling $W$ of $M$ an element of $\pi_{1}(W)$ of order strictly less than $k$.

Proof. We follow the lines of the proof of Theorem 1 but several details have to be adjusted to the new situation. To find a Bishop family around the binding $B$, we will construct a model around the binding $\widetilde{B}$ in the cover, perform all steps as in Theorem 1 and finally show that $\pi: \widetilde{N} \rightarrow N$ induces similar results in the base.

Note that the fundamental group $G:=\pi_{1}(N)$ acts by deck transformations on $\widetilde{N}$, and that $\tilde{N} / G \cong N$. We will identify a tubular neighborhood of $N$ in $M$ with a neighborhood $U$ of the 0 -section in the normal bundle $\nu N$. The universal cover of $\nu N$ is just given by the pull-back bundle $\pi^{-1}(\nu N)$ over $\widetilde{N}$, and so we find a neighborhood $\widetilde{U}$ of the 0 -section of $\pi^{-1}(\nu N)$ such that $\widetilde{U} / G=U$. We can also pull-back the contact form $\left.\alpha\right|_{U}$ to a $G$-invariant contact form $\widetilde{\alpha}$ on $\widetilde{U}$.

The contact form $\widetilde{\alpha}$ induces on $\widetilde{N}$ an open book decomposition, and in principle we can use [Nie06, Section 3] to obtain a neighborhood of the binding $\widetilde{B}$ strictly contactomorphic to a neighborhood of the 0 -section in $\mathbb{R}^{3} \times T^{*} \widetilde{B}$ with the contact form $d z+\frac{1}{2}(x d y-y d x)+\lambda_{\text {can }}$. We need to be a bit more careful though, because $\widetilde{B}$ does not need to be compact. But the construction of this contactomorphism is based on the Moser trick, and a closer inspection of the proof shows that not only does this contactomorphism exist, but that it is even $G$-equivariant: where $G$ acts on the $T^{*} \widetilde{B}$-factor by the linearization of the $G$-action on $\widetilde{B}$, and on the $\mathbb{R}^{3}$-factor by linear transformations leaving the $z$-direction invariant.

The symplectization $\mathbb{R} \times \widetilde{U}$ is the universal cover of the symplectization $\mathbb{R} \times U$. The fundamental group $G=\pi_{1}(N)$ acts trivially on the $\mathbb{R}$-factor, and thus respects the symplectic form $d\left(e^{t} \widetilde{\alpha}\right)$. It is also not difficult (though tiresome) to check that the almost complex structure $J$ constructed in [Nie06, Section 3] is also $G$-invariant.

As in the Proof of Theorem 1 we find in $\mathbb{R} \times \widetilde{U}$ a Bishop family of $J$-holomorphic disks, and also the corresponding family of codimension 2 almost complex submanifolds $S_{z}$ used for the intersection argument. Furthermore since $J$ is $G$-invariant, it follows that $G$ maps each of these families into itself, and so we may project the almost complex structure $J$, and these families into the symplectization $\mathbb{R} \times M$.

The boundary of the Bishop disks in $\mathbb{R} \times M$ intersect each page of the covered open book exactly $k$-times, and we supposed that $N$ is nucleation free so that bubbling is not possible. This way the rest of the proof is now exactly as the one of Theorem 1. 


\section{EXAMPLES AND APPLICATIONS}

The main difficulty consists in finding a situation where we can apply Theorems 1 and 2 to prove the Weinstein conjecture.

Note that it is easy to find examples of submanifolds with an induced Legendrian open book in any Darboux chart. For example, it is easy to see that $\mathbb{S}^{n+1}$ can be embedded into $\left(\mathbb{S}^{2 n+1}, \xi_{0}\right)$ via

$$
\left(x_{0}, \ldots, x_{n+1}\right) \in \mathbb{S}^{n+1} \hookrightarrow\left(x_{0}+i x_{1}, x_{2}, \ldots, x_{n+1}\right) \in \mathbb{C}^{n+1}
$$

such that the standard contact form restricts to $x_{0} d x_{1}-x_{1} d x_{0}$ which clearly defines the canonical open book on $\mathbb{S}^{n+1}$ with an $n$-ball as a page, and with trivial monodromy. Another example was given in [Nie06, Section 5.2], where it was shown that we can embed $\mathbb{S}^{2} \times \mathbb{S}^{n-1}$ in the desired way into $\left(\mathbb{R}^{2 n+1}, \xi_{0}\right)$.

On the other hand there are often evident obstructions to the realization of a homology class by a maximally foliated submanifold as an open book. For example, the only closed 2-dimensional manifolds that admit a proper or a covered open book decomposition are $\mathbb{S}^{2}$ and $\mathbb{R} \mathrm{P}^{2}$. The reason for this is that if $\Sigma$ is a closed surface that admits a (covered) open book, then we can lift the rotational vector field $\partial_{\varphi}$ from $\mathbb{S}^{1}$ to $\Sigma$, and obtain a vector field whose index is positive at each of its singularities. By the Poincaré-Hopf theorem it follows that the Euler characteristic of $\Sigma$ has to be positive, but the only compact surfaces that have positive Euler characteristic are $\mathbb{S}^{2}$ and $\mathbb{R P}^{2}$. Hence for purely topological obstructions, we obtain that $\mathbb{T}^{3}$ (or for example a hyperbolic 3 manifold) does not contain any embedded non-nullhomologous 2-sphere or real projective 2-space, because both would have to lift to a non-nullhomologous $\mathbb{S}^{2}$ in $\mathbb{R}^{3}$.

But it is also easy to give contact topological obstructions, because there are many contact manifolds that do not have contractible Reeb orbits as the following examples will show.

Example 3. Let $(M, \xi)$ be the unit cotangent bundle $\mathbb{S}\left(T^{*} \mathbb{T}^{n}\right)$ of the torus with its canonical contact structure. We can identify $M$ with $\mathbb{T}^{n} \times \mathbb{S}^{n-1}$ with coordinates $\left(x_{1}, \ldots, x_{n}\right) \in \mathbb{T}^{n}$ and $\left(y_{1}, \ldots, y_{n}\right) \in \mathbb{S}^{n-1}$ and write the canonical 1 -form as

$$
\lambda_{\text {can }}=\sum_{j=1}^{n} y_{j} d x_{j} .
$$

The Reeb field for this form is $R=\sum_{j} y_{j} \partial_{x_{j}}$, and so it follows that the orbits move in constant direction along the torus, and hence there will not be any closed contractible Reeb orbits. In particular it follows that it is not possible to embed any manifold with a Legendrian open book into $\left(\mathbb{S}\left(T^{*} \mathbb{T}^{n}\right), \lambda_{\text {can }}\right)$ that represents a non-trivial class in $H_{n+1}\left(\mathbb{S}\left(T^{*} \mathbb{T}^{n}\right), \mathbb{Z}_{2}\right)$.

After having described some of the problems of our method, we will give some positive examples.

Example 4. Let $(M, \xi)$ be a contact manifold that is subcritically Stein fillable, that means it can be filled by a Stein manifold of the form $(\mathbb{C} \times W, d x \wedge d y+d \lambda)$, where $(W, d \lambda)$ is a $2 n-$ dimensional Stein manifold. Then it follows that $(M, \xi)$ admits an open book with page $W$ and trivial monodromy consisting of taking the angular coordinate on the $\mathbb{C}$-factor of $\mathbb{C} \times W$ as a fibration over $\mathbb{S}^{1}$.

Any properly embedded Lagrangian submanifold $L$ in $W$ gives rise to an $(n+1)$-submanifold $N$ of $M$ that is foliated as a Legendrian open book. In fact, $N$ is obtained by taking the intersection of $\mathbb{C} \times L \subset \mathbb{C} \times W$ with the convex boundary $M$. Another way to describe the construction is by saying that we take the product of $L$ with $\mathbb{S}^{1}$, and then close this off by adding $\partial L \times \mathbb{D}^{2}$ in a neighborhood of the binding of $M$.

Unfortunately this manifold will often be homologically trivial. We can avoid this problem if $W$ is a Stein manifold with plurisubharmonic Morse function $h: W \rightarrow[0, \infty)$, and if the highest critical point $p_{0}$ is of index $n$, because then we can take for $L$ the unstable manifold of $p_{0}$ which will be a Lagrangian plane which intersects the skeleton of $W$ only in $p_{0}$. This way, we obtain for $N$ a sphere with the standard Legendrian open book decomposition, and the intersection between $N$ and the skeleton of any page is 1 , so that $[N]$ may not be trivial in $H_{n+1}\left(M, \mathbb{Z}_{2}\right)$, and we can apply our theorem to find a contractible Reeb orbit. 
The easiest examples that fit into this situation are unit bundles of $\mathbb{C} \oplus T^{*} S$ for any closed manifold $S$. To be even more explicit, take the contact structure $\xi$ on $\mathbb{T}^{n} \times \mathbb{S}^{n+1}$ given by

$$
\xi=\operatorname{ker}\left(\sum_{j=1}^{n} y_{j} d x_{j}+\frac{1}{2}\left(y_{n+1} d y_{n+2}-y_{n+2} d y_{n+1}\right)\right)
$$

with $\left(x_{1}, \ldots, x_{n}\right)$ the coordinates on $\mathbb{T}^{n}$, and $\left(y_{1}, \ldots, y_{n+2}\right)$ the coordinates on $\mathbb{S}^{n+1}$. Here, any sphere $\{\mathbf{x}\} \times \mathbb{S}^{n+1}$ is foliated by a Legendrian open book, and we obtain, in contrast to Example 3 . that $\left(\mathbb{T}^{n} \times \mathbb{S}^{n+1}, \xi\right)$ always has a closed contractible Reeb orbit.

Similarly the contact structure on $\mathbb{S}^{n} \times \mathbb{S}^{n+1}$ given by using the trivial open book with page $T^{*} \mathbb{S}^{n}$ and trivial monodromy also always has a closed contractible Reeb orbits.

Example 5. The most obvious example, where we find a submanifold with a Legendrian covered open book is the real projective space with the standard contact structure

$$
\left(\mathbb{R P}^{2 n-1}=\left\{\left[x_{1}: \cdots: x_{n}: y_{1}: \cdots: y_{n}\right] \mid \sum_{j}\left(x_{j}^{2}+y_{j}^{2}\right)=1\right\}, \quad \xi_{0}:=\operatorname{ker} \sum_{j=1}^{n}\left(x_{j} d y_{j}-y_{j} d x_{j}\right)\right)
$$

given as the quotient of the standard contact sphere $\mathbb{S}^{2 n-1}$ by the antipodal map. The submanifold $\left\{\left[x_{1}: \cdots: x_{n}: x_{n+1}: 0: \cdots: 0\right]\right\} \cong \mathbb{R} \mathrm{P}^{n+1}$ represents the non-trivial class in $H_{n}\left(\mathbb{R P}^{2 n-1}, \mathbb{Z}_{2}\right)$, and carries the covered open book described in Example 2. It follows from Theorem 2 that any contact form for $\xi_{0}$ admits a contractible closed Reeb orbit.

Note that the Weinstein conjecture for Example 5 is well known, because it has already been proved a long time ago for the standard contact structure on the unit sphere Rab78. Similarly the Weinstein conjecture for subcritically fillable manifolds can be proved in general (that means without imposing the condition on the critical points) by using the technically much more difficult results from SFT Yau04.

Still, we believe that our results have some value in depending only on local information, for example it is easy to prove:

Lemma 1. Let $\left(M_{1}, \xi_{1}\right)$ and $\left(M_{2}, \xi_{2}\right)$ be two closed cooriented contact manifolds with $\operatorname{dim} M_{1}=$ $\operatorname{dim} M_{2}$. If $\left(M_{1}, \xi_{1}\right)$ satisfies the conditions of Theorem 1 or 2 , then any contact form on the connected sum

$$
\left(M_{1} \# M_{2}, \xi_{1} \# \xi_{2}\right)
$$

has a contractible Reeb orbit.

Similar results can be obtained for other surgeries, but they require a more careful analysis in each situation.

\section{REFERENCES}

[AH09] P. Albers and H. Hofer, On the Weinstein conjecture in higher dimensions, Comment. Math. Helv. 84 (2009), no. 2, 429-436.

[Eps66] D. Epstein, The degree of a map, Proc. London Math. Soc. (3) 16 (1966), 369-383.

[Hof93] H. Hofer, Pseudoholomorphic curves in symplectizations with applications to the Weinstein conjecture in dimension three, Invent. Math. 114 (1993), no. 3, 515-563.

[MNW] P. Massot, K. Niederkrüger, and C. Wendl, Weak fillability of higher dimensional contact manifolds, in preparation.

[Nie06] K. Niederkrüger, The plastikstufe - a generalization of the overtwisted disk to higher dimensions., Algebr. Geom. Topol. 6 (2006), 2473-2508.

[NP10] K. Niederkrüger and F. Presas, Some remarks on the size of tubular neighborhoods in contact topology and fillability, Geom. Topol. 14 (2010), no. 2, 719-754.

[Rab78] P. Rabinowitz, Periodic solutions of Hamiltonian systems, Comm. Pure Appl. Math. 31 (1978), no. 2, $157-184$.

[Yau04] M.-L. Yau, Cylindrical contact homology of subcritical Stein-fillable contact manifolds., Geom. Topol. 8 (2004), 1243-1280.

E-mail address, K. Niederkrüger: niederkr@math.univ-toulouse.fr 
(K. Niederkrüger) Institut de mathématiques de Toulouse, Université Paul Sabatier - Toulouse III, 118 route de Narbonne, F-31062 Toulouse Cedex 9, France

E-mail address, A. Rechtman: rechtman@math.northwestern.edu

(A. Rechtman) Department of Mathematics, Northwestern University, 2033 Sheridan Road, Evanston, IL 60208-2730, United States of America 